Original Paper http://ajol.info/index.php/ijbcs http://indexmedicus.afro.who.int

\title{
Identification des insectes, parasites et évaluation économique de leurs pertes en graines sur les variétés améliorées et locale de niébé en milieu paysan à Karma (Niger)
}

\author{
Oumarou Haladou ISSOUFOU ${ }^{1 *}$, Soumana BOUBACAR ${ }^{2}$, Toudou ADAM ${ }^{1}$ \\ et Yamba BOUBACAR ${ }^{4}$ \\ ${ }^{1}$ Laboratoire de Productions Végétales, Faculté d'Agronomie, \\ Université Abdou Moumouni de Niamey, BP 10960 Niamey, Niger. \\ ${ }^{2}$ Département de Sociologie et Economie Rurales, Faculté d'Agronomie, \\ Université Abdou Moumouni de Niamey, BP 10960 Niamey, Niger. \\ ${ }^{3}$ Département de Géographie, Faculté des Lettres et des Sciences Humaines, \\ Université Abdou Moumouni de Niamey, BP 418 Niamey, Niger. \\ *Auteur correspondant, E-mail : isfouhaladou@yahoo.fr, Tel : (00227) 96460819.
}

\section{REMERCIEMENTS}

Les auteurs remercient l'ONG Comité d'Appui au Développement Local (CADEL) et le Programme de Productivité Agricole en Afrique de l'Ouest (PPAAO) pour le financement de la présente étude.

\section{RESUME}

Au Niger, la production de niébé est affectée par des contraintes biotiques et abiotiques. Les objectifs de la présente étude sont d'identifier les insectes et parasites au cours de la phénologie des variétés améliorées et locale de niébé ; d'estimer les pertes en graines et en valeur monétaire dues aux ravageurs. Les données ont été collectées à travers un suivi des parcelles paysannes. L'échelle standard de William (1984) a été utilisée pour les calculs de gravités d'attaques et de pertes. L'analyse de la variance (ANOVA) a été utilisée pour cette étude. Les résultats montrent que les parcelles de niébé étudiées ont enregistré les attaques de 8 insectes et 7 parasites. Ces ravageurs sont abondants à tous les stades du cycle de la culture. La variété locale a enregistré significativement la plus grande gravité d'attaque (66,25\% pour les insectes et $69,43 \%$ pour les parasites). La variété IT98K-205-8 a connu la plus faible gravité (12,35\% pour les insectes et 16,36\% pour les parasites). La plus grande perte $(355,42 \mathrm{~kg} / \mathrm{ha}$, soit $142168 \mathrm{FCFA})$ a été notée au niveau de la variété locale et la plus petite perte $(99,14 \mathrm{~kg} / \mathrm{ha}$, soit 39656 FCFA) a été enregistrée chez la variété IT98K-205-8.

(C) 2017 International Formulae Group. All rights reserved.

Mots clés : Niébé, contraintes de production, perte économique, Niger. 


\title{
Identification of insects, parasites and economic assessment of their grains losses on improved and local varieties of cowpea on farmers' field in Karma (Niger)
}

\begin{abstract}
In Niger, cowpea production is affected by biotic and abiotic constraints. The objectives of this study are to identify insects and parasites during the phenology of improved and local varieties of cowpea and to appraisal grains and monetary value losses due to pests. The data were collected through a monitoring of farmers' fields. William's standard scale (1984) was used to calculate the gravity of attacks and losses. ANOVA was used for data statistical analysis. The results showed that the plots of the cowpea used recorded the attacks of 8 insects and 7 parasites. These pests are abundant at all stages of the cowpea cycle. The local variety recorded significantly the greatest attack severity $(66.25 \%$ for insects and $69.43 \%$ for parasites). The IT98K-205-8 variety had the lowest severity (12.35\% for insects and $16.36 \%$ for parasites). The greatest loss ( $355.42 \mathrm{~kg} / \mathrm{ha}$, or $142168 \mathrm{FCFA})$ was observed on the local variety and the smallest loss $(99.14 \mathrm{~kg} / \mathrm{ha}$, or 39 656 FCFA) was recorded on IT98K-205-8 variety.

(C) 2017 International Formulae Group. All rights reserved.
\end{abstract}

Keywords: cowpea, production constraints, economic loss, Niger.

\section{INTRODUCTION}

En Afrique de l'Ouest, le niébé est une légumineuse qui participe à la sécurité alimentaire et à la réduction de la pauvreté (Allogni et al., 2004). Cependant, plusieurs contraintes biotiques et abiotiques s'opposent à la production et la rentabilité de cette culture. En effet, à chaque phase de sa croissance, le niébé est sévèrement attaquée par une multitude d'insectes, parasites et maladies (Dugjé et al., 2009 ; N'gbesso et al., 2013). Les dégâts se traduisent par la destruction de la plante, des dépréciations de la qualité des graines voire leur perte totale (Habiba, 2004; Talekar, 2006; Tonessia et al., 2009). Des nombreuses études prédisent d'importantes répercussions d'attaques des insectes et parasites sur la production et le revenu engendré par la production de niébé (Liao et Lin, 2000; Tamo et al., 2003; Habiaba, 2004 ; Talekar, 2006 ; Tonessia et al., 2009). Mais ces études supposent que les producteurs n'adoptent pas des stratégies efficaces de prévention ou de lutte contre ces ravageurs du niébé.

$\mathrm{Au}$ Niger, les insectes et parasites représentent les contraintes biotiques les plus importantes de la culture du niébé surtout dans un contexte caractérisé par des aléas climatiques. En effet, les attaques de ces insectes et parasites conduisent souvent simultanément à la réduction de la croissance ou à des pertes des plantes atteintes (Alavo, 2010; N'Gbesso et al., 2013; Yoka et al., 2014). Ces ravageurs infligent des dégâts très importants si aucune mesure de protection n'est mise en œuvre (Dugjé et al., 2009; Tonessia et al., 2009). Les principaux ravageurs de niébé détruisent les feuilles, les tiges, les fleurs, les gousses et les graines. Ces risques de pertes importantes de production sont les causes de démotivation des agriculteurs pour la culture de niébé (Tamo et al., 2003 ; Habiba, 2004).

Dans ce contexte, la rentabilisation et la pérennisation de la culture de niébé passent par une bonne gestion des ravageurs. Pour 
atteindre ces objectifs, plusieurs travaux de recherche ont porté sur l'identification et la caractérisation des insectes, parasites et maladies de niébé. Mais ces études abordent très peu la quantification des dégâts de ces ravageurs au cours de la phénologie de cette culture. Le présent travail cherche à contribuer à ce front de recherche. Il vise un double objectif: identifier d'abord les insectes ravageurs, les parasites et les maladies au cours de la culture de deux variétés améliorées de niébé et une variété locale et enfin estimer les pertes en graines occasionnées par ces ravageurs.

\section{MATERIEL ET METHODES} Zone d'étude

L'étude s'est déroulée dans le village de Karma, chef-lieu de la commune dans le département de Kollo en région de Tillabéry au Niger. Le village de Karma se situe à 35 Km à l'Ouest de Niamey, la capitale du Niger. Les coordonnées géographiques du site sont $13^{\circ} 40^{\prime} 299^{\prime \prime}$ de longitude et $001^{\circ} 48^{\prime} 772^{\prime \prime}$ de latitude. La commune rurale de Karma est située entre les longitudes $2^{\circ} 10$ Ouest et $1^{\circ} 40$ Est et les latitudes $18^{\circ} 5$ Nord et $13^{\circ} 33$ Sud. Elle s'étend sur une superficie d'environ 1313 $\mathrm{km}^{2}$. Peuplée d'environ 62247 habitants, la population majoritairement Zarma est concentrée sur la partie du fleuve Niger. Cette zone correspond à la zone sahélienne du Niger où la pluviométrie varie de $300 \mathrm{~mm}$ à $600 \mathrm{~mm}$ par an. Pour la campagne agricole 2013-2014, la moyenne des précipitations enregistrées était de $537 \mathrm{~mm}$. L'agriculture et l'élevage constituent les principales activités de la population de cette zone. Les argilolimoneuses, les glacis et les hydromorphes sont les types de sols les plus dominants du site. D'altitude 196 mètres (643 pieds), Karma a un climat de type sahélo-soudanien des précipitations variant entre 500 à $600 \mathrm{~mm}$ par an. Le choix du village de Karma est basé sur son accessibilité, la production de deux variétés améliorées de niébé dans un champécoles paysan et des champs de multiplication de semences. Le champ-écoles paysan, les champs de multiplication de semences et le champ témoins suivis ont chacun une superficie de 0,5 ha. Ces critères ont été validés grâce à des entretiens préliminaires conduits avec des agents communaux de l'Agriculture.

\section{Matériel végétal}

Le matériel végétal est constitué de deux variétés améliorées de niébé : IT97K499-35 et IT98K205-8 et de la variété locale la plus cultivée appelée Doungouri kwarey, ce qui signifie en Zarma niébé blanc. Les variétés IT97K499-35 et IT98K205-8 diffusées par le projet dans la zone de Karma proviennent de l'Institut National de la Recherche Agronomique du Niger (INRAN).

\section{Matériel technique}

Le matériel utilisé dans le cadre de cette étude été constitué de :

- un site d'essai, communément appelé champ école, qui sert à l'expérimentation de ces deux nouvelles variétés, mise en place par les paysans et supervisé par des techniciens ;

- deux sites de multiplication de semences dirigés par les paysans ;

- un site témoin pour la culture d'une variété locale, dirigé aussi par les paysans ;

- des pinces souples pour le prélèvement et le ramassage des insectes ;

- des tubes plastiques de $1,5 \mathrm{~cm}$ de diamètre et de $9 \mathrm{~cm}$ de hauteur contenant de l'alcool à $70{ }^{\circ} \mathrm{C}$ pour conserver les échantillons d'insectes ;

- des aiguilles pour piquer les insectes ;

- une loupe manuelle pour l'identification des pucerons et autres insectes non visibles à l'œil nu ; 
- une clé d'identification des insectes et parasites et autre matériel nécessaire pour la collecte de l'information.

Inventaire des insectes, maladies et parasites

Les observations ont été faites durant la campagne d'hivernage 2013-2014 sur les différents organes et stades phénologiques des variétés de niébé étudiées. Ces observations ont porté sur les insectes, les maladies et les parasites, leurs symptômes présents sur les plants et le nombre de plants attaqués dans le carré de rendement. Ces données ont été complétées par des observations et des collectes faites dans les exploitations familiales des environs de Karma. Ces observations ont permis de déterminer la présence de tous les signes d'origine pathologique et entomologique.

Estimation des pertes dues aux insectes, maladies et parasites

Les données issues des observations ont servi à l'estimation des dégâts par insectes, par parasites et maladies, par stades phénologiques, par variété et par sites. La méthode de calcul de pertes de l'échelle standard de William (1984) utilisée a comme formules :

- Formule de calcul de la gravité des attaques dans une parcelle

$S(\%)=\frac{\sum_{i}^{n}(X 1-1) N i \times 100}{[E(X 1)-1] \times N}$

Avec $\mathbf{S}$ : Gravité moyenne de la maladie dans la parcelle, X1 : catégories des échelles de notation, $\mathbf{X 1}=1$ à $5, \mathbf{E}(\mathbf{X 1})$ : étendue de l'échelle, donc 5 pour les insectes, $\mathbf{N i}$ : effectif de la catégorie $\mathrm{X} 1, \mathbf{N}$ : nombre total de plants

$P G(\mathrm{~kg})=\frac{S e \times R d \times S u}{100 \times 10}$

Où PG: perte en graines, Se : gravité moyenne (\%), Rd: rendement moyen en kg.ha ${ }^{1}, \mathbf{S u}$ : superficie (ha).
PM (FCFA) $=$ PG X P

Avec PM : Perte monétaire, PG : Perte en grain et $\mathbf{P}$ : Prix du $\mathrm{kg}$ au producteur

\section{Traitement des données}

Les analyses statistiques des données ont été réalisées en utilisant le logiciel XLSTATS version 13. Les procédures utilisées ont été l'analyse de la variance (ANOVA) et la séparation des moyennes par le test de Student Newman-Keuls (SNK) au seuil de signification de $5 \%$.

\section{RESULTATS}

Principaux insectes, maladies et parasites inventoriés selon la phénologie du niébé

Les informations sur l'identification des principaux insectes selon la phénologie du niébé sont présentées dans le Tableau 1. Des huit (8) espèces d'insectes inventoriées, trois (3) ont été observées uniquement sur la variété IT97K499-35 et locale (Empoasca spp par exemple) et les cinq (5) autres sont constatées sur toutes les trois (3) variétés (Megalurothrips sjostedti par exemple). La croissance végétative, la floraison et la fructification sont les trois (3) phases phénologiques où les insectes ont été les plus présents. Certains insectes ont été présents sur une seule phase (croissance végétative pour Empoasca spp et floraison pour Megalurothrips sjostedti et Mylabris senegalensis, Coryna argenteata et Decapotoma) alors que d'autres ont été observés sur deux (2) phases (croissance végétative et floraison pour Bemisia tabaci (Genn) et Alphis crassivora; floraison et fructification-maturation pour Mirperus jaculus, Acanthomia tomentosicollis et Anaplocnemis curvlpes), sur trois (3) phases (Spodoptera littoralis et Amsacta moloneyi Drc).

Huit (8) parasites et maladies ont été identifiés au cours de la culture des variétés étudiées (Tableau 2). Comme dans le cas des 
insectes, la croissance végétative, la floraison et la fructification sont les trois (3) phases phénologiques où les parasites et les maladies ont été les plus présents. Certains parasites et maladies ont été observés uniquement sur la variété locale (Striga gesuerioides), d'autres sur la variété IT97K499-35 et la variété locale (Cercospora canescens, Virus de CAbMV, Mosaïque jaune et Xanthomonas vignila) et d'autres sur les trois (3) variétés (IT98K205-8, IT97K499-35 et la variété locale) étudiées (Macrophotnina phaseolina et Phakopsora pachyrhizi). Certains parasites et maladies sont présents sur les phases croissance végétative et floraison (Cercospora canescens, Virus de CAbMV, Mosaique jaune, Xanthomonas vignila) et d'autres sur les trois (3) phases (Macrophotnina phaseolina, Phakopsora pachyrhizi et Striga gesnerioides).

Evaluation des pertes dues aux attaques des insectes, maladies et parasites

Gravité d'attaques des insectes sur les variétés étudiées

Les données sur la gravité d'attaques des insectes sur les variétés étudiées sont consignées dans le Tableau 3. Quel que soit le stade phénologique considéré, la variété locale a enregistré plus de gravité d'attaques dues aux insectes. Ensuite vient la variété IT97K499-35 et enfin la variété IT98K-205-8. Cette gravité d'attaques des insectes est plus importante en champ de multiplication de semences qu'en champ école paysan pour les variétés IT97K-499-35 et IT98K-205-8. Quelle que soit la variété de niébé considérée, la gravité diminue des feuilles aux gousses en passant par les fleurs.

Gravité d'attaques des maladies et parasites sur les variétés étudiées

Pour toutes les variétés, la gravité d'attaques des parasites et maladies augmente de la levée à la maturation. Quel que soit le stade phénologique considéré, la variété locale a enregistré plus de gravité d'attaques dues aux parasites et maladies. En champ école et en champ de multiplication de semence, c'est la variété IT97K-499-35 qui a connu plus de gravité d'attaques des parasites et maladies quel que soit le stade phénologique considéré. Cette gravité est aussi plus importante en champ de multiplication des semences qu'en champ école paysan aussi bien pour la variété IT97K-499-35 et pour la variété IT98K-205-8 quel que soit le stade phénologique. Ces informations sont présentées dans le Tableau 4.

\section{Pertes à l'hectare en grain et en valeur monétaire dues aux attaques des insectes, maladies et parasites}

Le Tableau 5 résume les données sur les pertes à l'hectare en grains et en valeurs monétaires des attaques des insectes au cours de la phénologie du niébé. Ces pertes sont statistiquement plus importantes chez la variété locale (Doungouri koiray) comparativement aux deux (2) variétés améliorées (IT97K-499-38 et IT98K-205-8). Quel que soit la variété et le site considéré, le niveau de perte en grains et en valeur monétaire diminue des insectes de feuilles aux insectes de gousses en passant par les insectes de fleurs. Ces pertes sont aussi plus importantes en champ de multiplication de semence qu'en champ école pour les variétés IT97K-499-38 et IT98K-205-8.

Le Tableau 6 présente les données sur les pertes à l'hectare en grains et en valeurs monétaires des attaques des parasites et maladies au cours de la phénologie du niébé. Comme pour les insectes, le même constat a été fait. En effet, ces pertes sont statistiquement plus importantes chez la variété locale (Doungouri koiray) comparativement aux deux (2) variétés améliorées (IT97K-499-38 et IT98K-205-8). Quels que soient la variété et le site considéré, le niveau de perte en grains et en valeur 
monétaire augmente de la levée à la maturation en passant par la croissance, la floraison et la fructification. Ces pertes sont aussi plus importantes en champ de multiplication de semence qu'en champ école chez les variétés IT97K-499-38 et IT98K-2058. Ainsi, de la lecture du Tableau 5, la gravité d'attaques des insectes tout comme celle des parasites a varié selon les variétés, les sites d'études et les stades phénologiques du niébé. Pour les insectes, on a constaté une diminution de la gravité en passant de la croissance végétative à la floraisonfructification quels que soient le site et la variété d'étude. La situation inverse est observée pour les parasites et maladies. Il faut noter que pour les trois stades phénologiques, c'est la variété locale qui a enregistré la plus grande gravité d'attaques aussi bien pour les insectes $(66,25 \%)$ à la phase croissance végétative, que pour les parasites et maladies $(69,43 \%)$ au stade fructification-maturation. La variété IT98K-205-8 a connu la plus faible gravité d'attaques des insectes $(12,35 \%)$ au stade fructification-maturation et des parasites et maladies $(16,36 \%)$ à la phase croissance végétative en champ école. Pour l'ensemble des agressions subies par les plants des variétés de niébé étudiées, la plus grande perte est constatée avec la variété locale. Cette perte est estimée à 335,46 kg/ha, soit 100638 FCFA pour les attaques des insectes. La perte est estimée à $355,42 \mathrm{~kg} / \mathrm{ha}$, soit 106620 FCFA pour les dégâts des parasites et maladies. La plus petite perte est notée avec la variété $I T 98 K-205-8$ en champ école paysan. Cette perte est de 99,14 kg/ha, soit 39656 FCFA pour les agressions des insectes. Elle est de 119,3 kg/ha, soit 47720 FCFA de perte occasionnée par les parasites et maladies. Ainsi les insectes, les parasites et maladies infligent de sérieux dégâts à la production en graines de niébé. Ce sont des quantités importantes de graines et de revenu de niébé qui sont perdues dues à des multiples agressions des insectes et parasites.

Tableau 1 : Insectes et leur présence selon les stades phénologiques du niébé.

\begin{tabular}{llc}
\hline Espèces & $\begin{array}{l}\text { Croissance } \\
\text { végétative }\end{array}$ & $\begin{array}{c}\text { Floraison } \\
\text { Maturation }\end{array}$ \\
\hline Empoasca spp $(++)$ & \\
Bemisia tabaci $(\mathrm{Genn})(++)$ & \\
Aphis crassivora $(++)$ & \\
Spodoptera littoralis et Amsacta \\
moloneyi Drc $(+++)$ \\
Megalurothrips sjostedti $(+++)$ \\
$\begin{array}{l}\text { Mylabris senegalensis, Coryna } \\
\text { argenteata } \text { et Decapotoma affinis } \\
(+++)\end{array}$ \\
Mirperus jaculus $(+++)$ \\
Acanthomia tomentosicollis et \\
Anaplocnemis curvlpes $(+++)$
\end{tabular}


Tableau 2 : Parasites et leur présence selon les stades phénologiques du niébé.

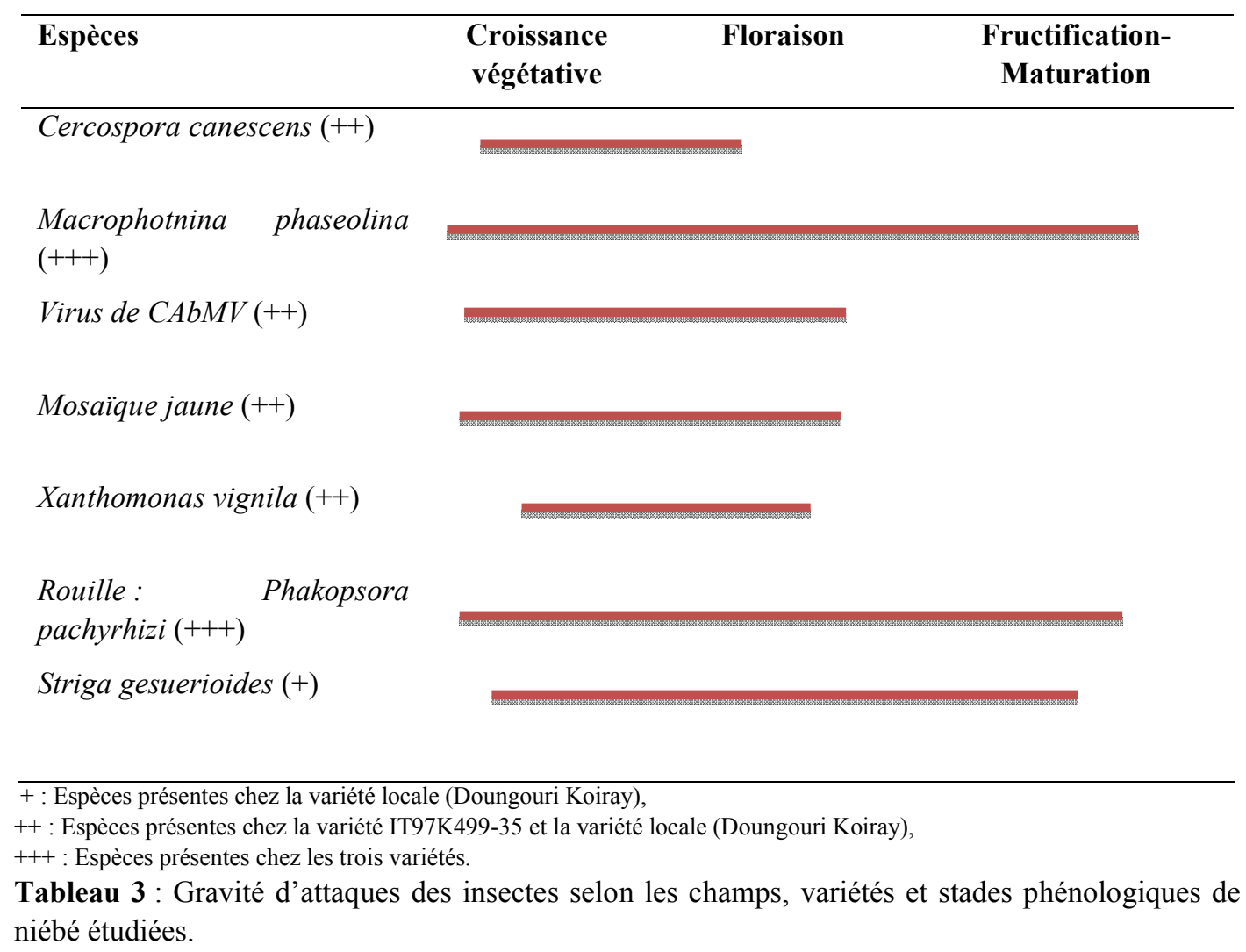

\begin{tabular}{|c|c|c|c|c|}
\hline \multirow[t]{3}{*}{ Champs } & \multirow[t]{3}{*}{ Variétés } & \multicolumn{3}{|c|}{ Gravité d'attaques dues aux insectes (\%) } \\
\hline & & Croissance & Floraison & Fructification- \\
\hline & & végétative & & Maturation \\
\hline \multirow[t]{2}{*}{$\mathrm{CE}$} & IT97K-499-35 & $22,5 \mathbf{b}$ & $19,77 \mathbf{b}$ & $16,7 \mathbf{b}$ \\
\hline & IT98K-205-8 & $21,66 \mathbf{b}$ & $15,56 \mathbf{b}$ & $12,35 \mathbf{b}$ \\
\hline \multirow[t]{2}{*}{ CMS } & IT97K-499-35 & $44,30 \mathbf{a}$ & $39,14 \mathbf{a}$ & $25,42 \mathbf{a b}$ \\
\hline & IT98K-205-8 & $38,43 \mathbf{a b}$ & $34,75 \mathbf{a b}$ & $20,63 \mathbf{a b}$ \\
\hline $\mathrm{CT}$ & « Doungouri Koiray » & $66,25 \mathbf{a}$ & $56,22 \mathbf{a}$ & $45,46 \mathbf{a}$ \\
\hline
\end{tabular}

Dans une même colonne, les valeurs affectées de la même lettre ne sont pas significativement différentes selon le test de Newman-Keuls (SNK) au seuil de probabilité de $5 \%$.

CE : Champ école ; CMS : Champ de multiplication de semences ; CT : Champ témoin. 
Tableau 4 : Gravité d'attaques des parasites selon les champs, variétés et stades phénologiques de niébé étudiées.

\begin{tabular}{llccc}
\hline Champs & Variétés & \multicolumn{3}{c}{ Gravité d'attaques dues aux parasites (\%) } \\
\cline { 3 - 5 } & & CV & F & FM \\
\hline CE & IT97K-499-35 & $19,22 \mathbf{b}$ & $23,10 \mathbf{b}$ & $26,35 \mathbf{~ b}$ \\
& IT98K-205-8 & $16,36 \mathbf{b}$ & $18,66 \mathbf{b}$ & $24,63 \mathbf{~ b}$ \\
\hline CMS & IT97K-499-35 & $39,45 \mathbf{a}$ & $42,48 \mathbf{a b}$ & $45,2 \mathbf{a b}$ \\
& IT98K-205-8 & $34,16 \mathbf{a b}$ & $38,6 \mathbf{a b}$ & $40,90 \mathbf{a b}$ \\
& & & & \\
\hline CT & « Doungouri Koiray » & $45,5 \mathbf{a}$ & $62,78 \mathbf{a}$ & $69,43 \mathbf{a}$
\end{tabular}

Dans une même colonne, les valeurs affectées de la même lettre ne sont pas significativement différentes selon le test de Newman-Keuls (SNK) au seuil de probabilité de 5\%.

CE : Champ école ; CMS : Champ de multiplication de semences ; CT : Champ témoin ; CV : Croissance végétative ; F : Floraison ; FM : Fructification-Maturation.

Tableau 5 : Perte à l'hectare en graine et valeur monétaire dues aux attaques des insectes selon les champs, variétés et stades phénologiques de niébé.

\begin{tabular}{|c|c|c|c|c|c|c|c|}
\hline \multirow[t]{2}{*}{ Champs } & \multirow[t]{2}{*}{ Variétés } & \multicolumn{4}{|c|}{ Pertes en graine $(\mathrm{Kg})$} & \multirow[b]{2}{*}{$\begin{array}{c}\text { Prix du Kg } \\
\text { (FCFA) }\end{array}$} & \multirow{2}{*}{$\begin{array}{c}\text { Pertes en valeur } \\
\text { monétaire } \\
\text { (FCFA) }\end{array}$} \\
\hline & & $\mathrm{CV}$ & $\mathbf{F}$ & FM & Total & & \\
\hline \multirow[t]{2}{*}{$\mathrm{CE}$} & IT97K-499-35 & 45 & 39,54 & 33,4 & 117,94 & 400 & 47176 \\
\hline & IT98K-205-8 & 43,32 & 31,12 & 24,7 & 99,14 & 400 & 39656 \\
\hline \multirow[t]{2}{*}{ CMS } & IT97K-499-35 & 88,6 & 78,28 & 50,84 & 217,72 & 400 & 87088 \\
\hline & IT98K-205-8 & 76,86 & 69,5 & 41,26 & 187,62 & 400 & 75048 \\
\hline \multirow[t]{2}{*}{$\mathrm{CT}$} & « Doungouri & 132,5 & 112,44 & 90,52 & 335,46 & 300 & 100638 \\
\hline & Koiray » & & & & & & \\
\hline
\end{tabular}


Tableau 6: Perte à l'hectare en graine et valeur monétaire dues aux attaques des parasites selon les champs, variétés et stades phénologiques de niébé.

\begin{tabular}{lllllllll}
\hline Champs & Variétés & \multicolumn{5}{c}{ Pertes en graine (Kg) } & & $\begin{array}{l}\text { Pertes en } \\
\text { valeur } \\
\text { monétaire } \\
\text { (FCFA) }\end{array}$ \\
\cline { 3 - 9 } & & CV & F & FM & Total & $\begin{array}{l}\text { Prix du } \\
\text { (FCFA) }\end{array}$ & Kg \\
\hline CE & IT97K-499-35 & 38,44 & 46,2 & 52,7 & 137,34 & 400 & 54936 \\
& IT98K-205-8 & 32,72 & 37,32 & 49,26 & 119,3 & 400 & 47720 \\
\hline CMS & IT97K-499-35 & 78,9 & 84,96 & 90,2 & 254,06 & 400 & 101624 \\
& IT98K-205-8 & 68,32 & 77,2 & 81,8 & 227,32 & 400 & 90928 \\
\hline CT & « Doungouri & 91 & 125,56 & 138,86 & 355,42 & 300 & 106620 \\
& Koiray » & & & & & & & \\
\hline
\end{tabular}

CE : Champ école ; CMS : Champ de multiplication de semences ; CT : Champ témoin ; CV : Croissance végétative ; F : Floraison ; FM : Fructification-Maturation.

\section{DISCUSSION}

Les résultats obtenus ont montré qu'au niveau des champs, toutes les trois variétés étudiées de niébé ont été exposées aux divers attaques des insectes et parasites principalement pendant la croissance végétative, la floraison et la fructificationmaturation. On peut ainsi admettre que ces phases sont de moments critiques pour la réussite de la culture de niébé. Ce résultat est en harmonie avec les conclusions de Habiba (2004) au Niger, Talekar (2006) en Tanzani, Dugjé et al. (2009) en Afrique de l'Ouest, Tonessia et al. (2009) au Sénégal, N'gbesso et al. (2013) en Côte d'Ivoire. En effet, dans leurs études, ces auteurs ont trouvé que la plante de niébé dans son écologie, se trouve confrontée à des multiples agressions des insectes et parasites. Les principaux insectes inventoriés par ces auteurs sont: Pucerons, Lagria, trips, Punaises, Bruches et Criquets. Les maladies se résument aux Viroses, Galles,
Eclatements des gousses et tâches de Rouilles sur les feuilles. Au Congo, Yoka et al. (2014) ont montré que parmi les paramètres susceptibles d'expliquer la diversité et l'abondance des ravageurs sur une culture, il convient de considérer les facteurs écologiques (climat, sol, environnement) et les pratiques culturales. En plus de ces facteurs, les ravageurs sont aussi favorisés par l'état physiologique du niébé (Habiba, 2004; Mahinto et al., 2014). En effet, le feuillage favorise la formation des nids de ponte puis de nourriture aux larves d'insectes. La présence des organes fructifères qui, par leur odeur, attirent également de nombreux insectes et virus de niébé dont ils sont les agents vecteurs (N'gbesso et al., 2013).

Les différents insectes, parasites et maladies ont induit des agressions diverses sur toutes les variétés de niébé étudiées. Le striga gesnerioides observé uniquement au niveau de la variété locale est une phanérogame parasite 
qui est très répandue dans les régions à faible pluviométrie et à sols peu fertiles. Sur les champs d'étude, cette plante parasite provoque le jaunissement des parties du limbe comprises entre les nervures, entraînant ainsi la mort des plants infestés et de pertes de rendement. En Afrique de l'Ouest, Dugjé et al. (2009) ont rapporté que le mal s'aggrave lorsque l'humidité du sol devient un facteur limitant. Ainsi affaiblies, les plants deviennent des cibles faciles aux infestations causées par les champignons et autres micro-organismes parasites. Les insectes de la préfloraison endommagent les plantules de niébé en prélevant la sève sur la face inférieure des jeunes feuilles, des jeunes tiges succulentes et des gousses des plantes arrivées à maturités. Ces ravageurs défoliateurs attaquent les limbes foliaires pour percer les feuilles de niébé de multiples trous. Les pullulations importantes des ravageurs défoliateurs ont pour effets des pertes de récoltes très conséquentes, voire totales. De son côté, Alavo (2010) a montré qu'au Bénin, les attaques sévères des insectes provoquent le rabougrissement de la plante, la déformation des feuilles, la défoliation précoce et le dépérissement des plantules. Nous avons aussi observé que les ravageurs des fleurs et des bourgeons floraux rongent fortement les pétales et les sépales vers le bas. Ces organes se fanent rapidement puis tombent au sol. Dans le même temps, les ovaires sont taraudés et les filets des étamines sont également détruits partiellement. Ces observations corroborent de celles Mehinto et al. (2014) et Habiba (2004) qui ont conclu que les chutes des fleurs interdisent les formations de gousses chez les niébé. Les insectes de la floraison/post-floraison s'installent sur les boutons floraux et sur les fleurs et se nourrissent de ces organes du niébé. Les plants gravement atteints ne produisent plus de fleurs. Ces observations vont dans le même sens que les résultats de Talekar (2006) en Tanzanie et N'gbesso et al. (2013) en Côte d'Ivoire. En effet, dans leurs études, ces auteurs ont montré que chez le niébé, lors d'attaques massives, les fleurs ouvertes sont déformées et décolorées, les boutons floraux et les fleurs tombent trop tôt et empêchent ainsi la formation des gousses. Avant la récolte, nous avons aussi remarqué que les ravageurs de gousses et des graines développent des piqûres qui ponctuent les gousses de multiples petits points noirs et provoquent l'apparition au niveau des graines atteintes des plissements et dessèchement des amandes avec formations plus ou moins rapidement de nécrose. Des études similaires ont expliqué que les foreuses de gousses se nourrissent des parties tendres des tiges, des pédoncules, des boutons floraux, des fleurs et des gousses encore fraîches, ce qui entraine la dépréciation du produit (Liao et Lin., 2000). Les punaises suceuses de gousses font beaucoup de dégâts sur le niébé. Ils piquent les gousses pour en sucer la sève et, ce faisant, entrainent leur dessèchement d'où une perte en semences (Talekar 2006; N'gbesso et al., 2013).

Face à ces différentes agressions des insectes, parasites et maladies, il va de soi que les variétés étudiées de niébé enregistrent des pertes significatives sur la production. Toutefois, il semble que les parasites causent plus de pertes que les insectes. Des résultats similaires ont été obtenus sur le niébé en Côte d'Ivoire par N'gbesso et al. (2013), au Bénin par Alavo (2010) et Mehinto et al. (2014) avec respectivement un taux d'attaques de 17 à $83 \%, 22$ à $60 \%$ et 17 à $66 \%$. Les pertes de 
rendements obtenus sont aussi comparables aux dégâts des insectes atteignant 80 à 100\% trouvés par Dugjé et al. (2009) en Afrique de l'Ouest. Les mêmes résultats ont été rapportés par Sarr et al. (2016) au Sénégal où ils ont constaté sur le cotonnier un taux d'attaque atteignant 50 à $70 \%$ en absence de traitement chimique. Les variations du niveau d'attaques des insectes, des parasites et des maladies ainsi que la perte de production entre les variétés de niébé pourraient s'expliquer par des différences au niveau des caractéristiques intrinsèques des variétés, des techniques culturales et des caractéristiques des sols (Habiba, 2004).

Ces insectes, parasites et maladies constituent donc, des très dangereux ravageurs pour le niébé. Les conséquences économiques réelles de leurs attaques seraient très dépendantes de l'état de développement atteint par les plantes concernées. Des méthodes de protection et de lutte contre les insectes, les parasites et maladies doivent être intensifiées durant les phases de croissance végétative, de floraison et de fructificationmaturation du niébé si l'on veut améliorer le taux de nouaison et réduire celui des avortements.

\section{Conclusion}

Au Niger, la culture de niébé connaît de sérieuses attaques des ravageurs. Dans cette étude, nous avons identifié les insectes, parasites et maladies et évaluer leurs pertes en graines et valeur monétaire à Karma. Il ressort de cette étude que les insectes, les parasites et les maladies de niébé sont plus abondants au cours des phases de croissance végétative, de floraison et de fructification-maturation. Certains de ces ravageurs sont observés sur une seule phase, d'autres sur deux, voire les trois phases critiques de cycle de la plante. Les gravités d'attaques varient selon les ravageurs, les variétés de niébé, les stades phénologques et les sites d'étude. La variété locale a affiché la plus grande gravité d'attaques et par conséquent la plus grande perte à l'hectare en graines et en valeur monétaire pour tous les ravageurs et les stades phénologiques. Mais même les semences améliorées qui sont censées être résistantes aux insectes, parasites et maladies ont connu d'attaques non négligeables surtout en champ de multiplication de semences. Les insectes, les parasites et les maladies infligent d'importants dégâts à toute la phénologie de niébé. Les pertes dues aux attaques des insectes, des parasites et des maladies réduisent ainsi la sécurité alimentaire et aggravent la pauvreté des producteurs de niébé. Des méthodes de protection et de lutte contre ces ravageurs doivent être intensifiées durant les phases de croissance végétative, de floraison et de fructification-maturation $\mathrm{du}$ niébé si l'on veut améliorer durablement le taux de nouaison et réduire celui des avortements. Aussi, pour maximiser l'impact et assurer la durabilité de la production, la diffusion des semences améliorées de niébé en milieu paysan doit être accompagnée par des mesures adéquates de protection et de lutte contre les ravageurs.

\section{CONFLIT D'INTERETS}

Les auteurs déclarent qu'il n'y a aucun conflit d'intérêt.

\section{CONTRIBUTIONS DES AUTEURS}

$\mathrm{OHI}$ a assuré la collecte, l'analyse des données, l'élaboration du projet d'article et la correction du manuscrit. SB et TA ont participé à l'analyse des données et la 
correction de l'article. YB a contribué à la rédaction du manuscrit.

\section{REMERCIEMENTS}

Les auteurs remercient l'Association Internationale des Sciences Agronomiques (AISA) pour l'organisation du congrès international à Yamoussoukro (Côte d'Ivoire) et tous ceux qui ont participé à ce travail (étudiants stagiaires, producteurs, services techniques, etc.). Enfin, les auteurs remercient vivement les deux examinateurs pour leurs précieuses suggestions d'amélioration de la qualité du manuscrit.

\section{REFERENCES}

Allogni WN, Coulibaly ON, Honlonkou AN. 2004. Impacts des nouvelles technologies duniébé sur la sécurité alimentaire des ménages agricoles au Bénin : Cas du département de l'Ouémé. Bulletin de la Recherche Agronomique du Bénin, 44 : 13-22. DOI : http://www.slire.net/download/802/articl e2_brab-44-2004_allogni_et_al_impactnouvelles_technologies-ni_b_s_curit_alimentaire-m_nages.pdf

Dugjé IY, Omoigui LO, Ekeleme F, Kamara AY, Ajeigbe H. 2009. Production du niébé en Afrique de l'Ouest : Guide du paysan. Document de l'IITA, p. 20.

Habiba Y. 2004. Evaluation agronomique de quelques variétés de niébé pour la production de graines et des fanes et leur résistance aux principaux ennemis. Mémoire de Maîtrise, Université de Niamey, Niger, p. 32.

Liao CT, Lin CS. 2000. Occurrence of legume podborer Maruca testulalis Geyer (Lepidoptera: Pyralidae) on cowpea Vigna unguiculata (L). Walp and its insecticides application trial. Plant Protec. Bul., 42: 213-222. DOI: http://farmer.iyard.org/pps/42/42-402.pdf

N'Gbesso FPM, Fondio L, Dibi BEK, Djidli HA, Kouame CN. 2013. Étude des composantes du rendement de six variétés améliorées de niébé [Vigna unguiculata (L.) Walp]. J. Apl. Biosc., 63: 4754 - $4762 . \quad$ DOI: https://www.ajol.info/index.php/jab/artic le/view/87249

N'gbesso MFP, Zohouri GP, Fondio L, Djidji AH, Konate D. 2013. Etude des caractéristiques de croissance et de l'état sanitaire de six variétés améliorées de niébé [Vigna unguiculata (L)] en zone centrale de Côte d'Ivoire. Int. J. Biol. Chem. Sci., 7(2): 457-467. DOI : http://dx.doi.org/10.4314/ijbcs.v7i2.4

Talekar N. 2006. Eléments de cours en défense des cultures: Pest management in soybean and cowpea cultivation. Treizième cours régional sur la recherche et la production des plantes légumières, 01 Juillet au 06 Octobre 2006 en Tanzanie, AVRDC, Arusha-Tanzania, p. 54.

Alavo TBC. 2010. Effet répulsif du kaolin contre les populations d'Aphis craccivora (Homoptera: Aphididae) sur le niébé (Vigna unguiculata). Int. J. Biol. Chem. Sci., 4(2): 407-414. DOI : https://www.ajol.info/index.php/ijbcs/art icle/view/58138

Mehinto JT, Atachi P, Elegbede M, Kpindou OKD, Tamo M. 2014. Efficacité comparée des insecticides de natures différentes dans la gestion des insectes ravageurs du niébé au centre Bénin. $J$. Apl. Biosc., 84: 7695-7706. DOI: 
https://www.ajol.info/index.php/jab/artic le/viewFile/112063/101826

Sarr M, Badiane D, Sane B. 2016. Evaluation de l'efficacité de nouveaux programmes de protection phytosanitaire contre les principaux ravageurs $\mathrm{du}$ cotonnier Gossypium hirsutum L. au Sénégal. Int. J. Biol. Chem. Sci., 10(5): 2163-2174. DOI:

http://dx.doi.org/10.4314/ijbcs.v10i5.18

Tamo M, Ekesi S, Maniania NK, Cherry A. 2003. Biological control non-obvious componentintegrated pest management for cowpea. In Biological Control in Integrated Pest Management Systems in Africa. Neuenschwander P, Borgemeister C, Langewald J. (eds). CAB International : Wallingford ; 205-309.

Tonessia C, Wade M, Cissé N, Aké S. 2009. Caractérisation de Striga gesnerioides
(Willd.) Vatke du Sénégal : Réactions de plusieurs cultivars de niébé (Vigna unguiculata L. Walp.). J. Apl. Biosc., 24: 1462-1476. DOI: http://www.m.elewa. org/JABS/2009/24/1.pdf

Williams RJ. 1984. Downy mildews of tropical cereals. In Advances in Plant Pathology, Ingram DS, Williams $\mathrm{PH}$ (eds). London Acad. Press: London; 1103.

Yoka J, Loumeto JJ, Djego JG, Houinato M, Akouango P. 2014. Adaptation d'un cultivar de Niébé (Vigna unguiculata (L.) Walp.) aux conditions pédoclimatiques de Boundji (République $\mathrm{du}$ Congo). Afrique SCIENCE, 10(1): 217- 225. DOI : http://www. afriquescience.info/document.php?id=32 30 